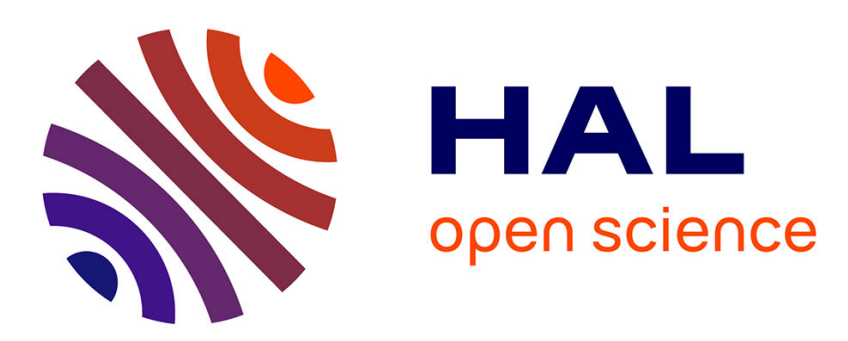

\title{
Partial amplitude synchronization detection in brain signals using Bayesian Gaussian mixture models
}

Maxime Rio, Axel Hutt, Bernard Girau

\section{To cite this version:}

Maxime Rio, Axel Hutt, Bernard Girau. Partial amplitude synchronization detection in brain signals using Bayesian Gaussian mixture models. Cinquième conférence plénière française de Neurosciences Computationnelles, "Neurocomp'10", Oct 2010, Lyon, France. pp.109 - 113. hal-00534076

\section{HAL Id: hal-00534076 \\ https://hal.science/hal-00534076}

Submitted on 8 Nov 2010

HAL is a multi-disciplinary open access archive for the deposit and dissemination of scientific research documents, whether they are published or not. The documents may come from teaching and research institutions in France or abroad, or from public or private research centers.
L'archive ouverte pluridisciplinaire HAL, est destinée au dépôt et à la diffusion de documents scientifiques de niveau recherche, publiés ou non, émanant des établissements d'enseignement et de recherche français ou étrangers, des laboratoires publics ou privés. 


\title{
Partial amplitude synchronization detection in brain signals using Bayesian Gaussian mixture models
}

\author{
Maxime Rio, Axel Hutt, Bernard Girau \\ Loria \\ Campus Scientifique - BP 239 - 54506 Vandoeuvre-lès-Nancy Cedex \\ \{maxime.rio, axel.hutt, bernard.girau\}@loria.fr
}

\begin{abstract}
In the last decade, the analysis of the synchronization between different brain signals has attracted much attention. In this context, detection methods of amplitude synchrony computed on time-frequency maps consider the baseline activity before stimulus onset. The present work introduces a new method to detect subsets of synchronized channels that do not consider any baseline information. It is based on a Bayesian Gaussian mixture model applied at each location of a time-frequency map. The work assesses the relevance of detected subsets by a stability measure.
\end{abstract}

\section{KEY WORDS}

Amplitude synchronization, Partial synchronization, Morlet wavelet, Gaussian mixture model, Mean-field approximation

\section{Introduction}

In neuroscience, synchronization mechanisms play an important role to explain underlying brain processes. The concept of synchronization itself has been defined in many different ways, leading to different methods to quantify it between different channels of an electrophysiological record (see [1, 2] for reviews).

In this article, we will focus on eventrelated synchronization (ERS) and event-related desynchronization (ERD) [3]. This kind of synchronization is defined as an increase or decrease, respectively for ERS and ERD, of the signal power in particular frequency bands, related to a stimulus or a response. It is typically quantified using averages over several trials of time-frequency power distributions, compared to some basis activity recorded before a stimulus onset. This data analysis approach has fruitfully shown ERS/ERD phenomena in alpha, beta and gamma bands for sensory and motor tasks.

As stated in [3], ERS/ERD quantities are computed as a percentage of power increase/decrease compared to some baseline activity. This approach is due to the overall decrease of power in high frequencies: phenomenons of interest at high frequencies have a much smaller amplitude than those at low frequencies. However, in a special case called partial synchronization such a normalization process is unnecessary to investigate relative ERS/ERD phenomenons.

We describe partial synchronization as synchronization of recorded channels within different subsets. Consequently, when a temporal epoch shows partial synchronization it is possible to segregate channels into several synchronized clusters. The proposed method performs such a clustering of channels at each time for a specific frequency band while selecting the number of relevant clusters through a Bayesian approach.

The following section describes the steps of the method:

1. the time-frequency representation applying wavelet analysis,

2. the clustering algorithm using a Bayesian Gaussian mixture model,

3. the stability analysis, used to quantify the spread in time and frequency of a cluster.

Section 3 presents the application of the method on an artificial test case and a real dataset.

\section{Method}

\subsection{Morlet wavelet}

In a first step, the signal $X^{(c)}(t)$ of each channel $c$ is decomposed into a time-frequency representation by a continuous wavelet transform (CWT), using a normalized Morlet wavelet.

Let $\psi(t)$ be a mother wavelet, daughter wavelets are defined as translations and dilatations of this mother wavelet:

$$
\psi_{a, \tau}(t)=\frac{1}{\sqrt{a}} \psi\left(\frac{t-\tau}{a}\right)
$$

where $a$ is the scale, and $\tau$ the shift. The CWT coefficients are computed by a convolution of the signal $X^{(c)}(t)$ with the daughter wavelets:

$$
W_{a, \tau}^{(c)}=\int X^{(c)}(t) \frac{1}{\sqrt{a}} \psi^{*}\left(\frac{t-\tau}{a}\right) d t
$$

where $^{*}$ denotes the complex conjugate.

A wavelet $\psi(t)$ localizes the signal within a time-frequency window $\left[t_{0} \pm \sigma_{t} ; f_{0} \pm \sigma_{f}\right]$, also called 
"Heisenberg box" [4]. With regards to the power of the wavelet, $t_{0}$ and $f_{0}$ are respectively the central time and frequency, $\sigma_{t}$ and $\sigma_{f}$ are the standard deviations in time and frequency:

$$
\begin{aligned}
t_{0} & =\int t|\psi(t)|^{2} d t \\
f_{0} & =\int f|\hat{\psi}(f)|^{2} d f \\
\sigma_{t} & =\int\left(t-t_{0}\right)^{2}|\psi(t)|^{2} d t \\
\sigma_{f} & =\int\left(f-f_{0}\right)^{2}|\hat{\psi}(f)|^{2} d f
\end{aligned}
$$

where $\hat{\psi}(f)$ is the Fourier transform of the wavelet.

For a daughter wavelet $\psi_{a, \tau}(t)$, time-resolution increases as frequency-resolution decreases with the scale, and the other way around. The Heisenberg box becomes $\left[\left(t_{0}-\tau\right) \pm a \sigma_{t} ; \frac{f_{0}}{a} \pm \frac{\sigma_{f}}{a}\right]$.

It is important to note that the maximum timefrequency resolution is bounded from below: $\sigma_{t} \sigma_{f} \geqslant \frac{1}{4 \pi}$.

The Morlet wavelet is extensively used to analyse electrophysiological signals. Its normalized version, a special case of the Gabor wavelet [5], is defined as follows:

$$
\psi(t)=\pi^{-\frac{1}{4}} e^{-\frac{t^{2}}{2}} e^{i \eta t}
$$

where $\eta$ is the number of cycles of the Morlet wavelet. This wavelet is well defined for $\eta>5$, and usually $\eta$ is chosen between 5 and 7 .

For the Morlet wavelet, $\sigma_{t}=\frac{1}{\sqrt{2}}$ and $\sigma_{f}=\frac{1}{2 \sqrt{2} \pi}$, which reach the theoretical time-frequency resolution bound [4].

More details about how to implement CWT with a Morlet wavelet are given in [6].

\subsection{Bayesian Gaussian mixture models}

Let $\quad \mathbf{w}=\left(\left|W_{a, \tau}^{(1)}\right|^{2}, \ldots,\left|W_{a, \tau}^{(c)}\right|^{2}, \ldots\right)$ be the vector containing the squared modulus of wavelet coefficients for all channels at some scale $a$ and shift $\tau$. Channels will be grouped using $\mathbf{w}$ as an input to a clustering algorithm.

More specifically, we use a Bayesian version of the Gaussian mixture model to do this clustering. Bayesian approaches exhibit several advantages compared to standard methods like, e.g., model comparison and automatic pruning of irrelevant clusters [7]

Let $\mathcal{W}=\left(\mathcal{W}_{1}, \ldots, \mathcal{W}_{c}, \ldots\right)$ be a set of random variables whose observations are w. A latent vectorial random variable $Z_{c}$ is associated to each $\mathcal{W}_{c}$, indicating from which Gaussian component it has been drawn. For $K$ Gaussian components, the conditional probability of $\mathcal{W}_{c}$ on $Z_{c}$ is written:

$$
p\left(w_{c} \mid z_{c}\right)=\Pi_{k=1}^{K} \mathcal{N}\left(w_{c} \mid \mu_{k}, \lambda_{k}^{-1}\right)^{z_{c, k}}
$$

where $z_{c}=\left(z_{c, 1}, \ldots, z_{c, K}\right)$ so that only one $z_{c, k}$ is equal to 1 , all the others are zero, and $\mathcal{N}$ denotes the Gaussian density function. In Eq. (5), $\mu_{k}$ and $\lambda_{k}$ are the mean and standard deviation of the corresponding density function. The density of the latent variables $Z_{c}$ is defined as follows:

$$
p\left(z_{c}\right)=\Pi_{k=1}^{K} \pi_{k}^{z_{c, k}} \text { with } \sum_{k=1}^{K} \pi_{k}=1
$$

where $\pi_{k}$ is the proportion of the $k$-th Gaussian component in the mixture.

The parameters of the model are $\mu_{k}, \lambda_{k}$ and $\pi_{k}$ for each Gaussian component $k$. In a Bayesian formulation, these parameters are treated as random variables, with a density function defined by hyperparameters.

A common choice for parameter distributions are conjugated distributions in order to keep further computations simple [7, 8]. In the present context assuming with an exponential model, it means that

- each $\mu_{k}$ follows a Gaussian distribution, $p\left(\mu_{k}\right)=$ $\mathcal{N}\left(\mu_{k} \mid m_{\mu}, \beta^{-1}\right)$

- each $\lambda_{k}$ follows a Gamma distribution, $p\left(\lambda_{k}\right)=$ $\mathcal{G}\left(\lambda_{k} \mid a_{\lambda}, b_{\lambda}\right)$

- and $\pi=\left(\pi_{1}, \ldots, \pi_{K}\right)$ follows a Dirichlet distribution, $p(\pi)=\operatorname{Dir}(\pi \mid \alpha)$.

Then Bayesian inference consists in computing the posterior probability of the model, which is the joint probability of the latent variables and parameters given the observed variables:

$p(z, \mu, \lambda, \pi \mid \mathbf{w})=\frac{\Pi_{c=1}^{C} p\left(w_{c} \mid \mu, \lambda, z_{c}\right) p(\mu) p(\lambda) p\left(z_{c} \mid \pi\right) p(\pi)}{p(\mathbf{w})}$

where $\mu=\left(\mu_{1}, \ldots, \mu_{K}\right), \lambda=\left(\lambda_{1}, \ldots, \lambda_{K}\right), z=\left(z_{1}, \ldots, z_{C}\right)$, and $p(\mathbf{w})$ the marginal probability of the model.

For mixture models, the posterior probability can not be computed exactly. To estimate $p(z, \mu, \lambda, \pi \mid \mathbf{w})$, we apply a deterministic approximation, called naive mean-field approximation or variational Bayes [7] [8]. The major idea is to approximate the joint posterior probability by a factorized distribution:

$$
p(z, \mu, \lambda, \pi \mid \mathbf{w}) \approx q(\pi) \Pi_{c=1}^{C} q\left(z_{c}\right) \Pi_{k=1}^{K} q\left(\mu_{k}\right) q\left(\lambda_{k}\right)
$$

This approximated posterior $q$ is made as close as possible to the real posterior probability $p$ by minimizing the Kullback-Leibler divergence $K L(q \| p)$. It is equivalent to maximizing the quantity $\mathcal{L}_{m}$ :

$$
\begin{aligned}
K L(q \| p) & =-\int q(v) \ln \frac{p(\mathrm{v}, \mathbf{w})}{q(\mathrm{v})} d v \\
\mathcal{L}_{m} & =\int q(v) \ln \frac{p(v \mid \mathbf{w})}{q(\mathrm{v})} d v
\end{aligned}
$$

where $v=(\pi, z, \mu, \lambda)$ represent all parameters and hidden variables of the model.

This leads to the approximated posterior functional form, which is a set of exponential distributions:

$$
\begin{aligned}
q\left(z_{c}\right) & =\Pi_{k=1}^{K} \tilde{\pi}_{k}^{z_{c, k}} \text { with } \sum_{k=1}^{K} \tilde{\pi}_{k}=1 \\
q\left(\mu_{k}\right) & =\mathcal{N}\left(\mu_{k} \mid \tilde{m}_{\mu_{k}},{\tilde{\beta_{k}}}^{-1}\right) \\
q\left(\lambda_{k}\right) & =\mathcal{G}\left(\lambda_{k} \mid \tilde{a}_{\lambda_{k}}, \tilde{b}_{\lambda_{k}}\right) \\
q(\pi) & =\mathcal{D i r}\left(\pi \mid\left(\tilde{\alpha}_{0}, \ldots, \tilde{\alpha}_{K}\right)\right)
\end{aligned}
$$

and an iterative procedure, like the E-M algorithm, with re-estimation equations used to optimize the hyperparameters of these distributions. 
Since, this algorithm provides convergence to a local maximum only several trials with random initializations are carried out. The quantity $\mathcal{L}_{m}$ is also a lower bound of the marginal probability $p(\mathbf{w})$. Consequently, it is computed to compare inferred posterior probabilities and select the best trial.

The whole inference procedure as been computed using the variational message passing algorithm (VMP), detailed in [9]. This algorithm is used to derive the reestimation equations as messages exchanged through a graphical representation of the probabilistic model.

The number of Gaussian components needed to model the data is automatically determined, with an automatic relevance determination mechanism [7]. Due to the Dirichlet distribution associated with the parameters $\pi$, irrelevant components are systematically pruned from the model. Moreover, models are initialized with a large number of components. A component $k^{\prime}$ to be discarded is not assigned to any point w.r.t the posterior probability:

$$
\sum_{c=1}^{C} \mathbb{E}_{q(z)}\left[z_{c, k^{\prime}}\right]<\varepsilon
$$

where $\varepsilon>0$ is chosen arbitrary small. In practice, one chooses $\varepsilon=1$, which means that, on average, pruned components are not even associated to one channel.

\subsection{Stability measure}

Since CWT is a redundant transform due to the strong correlation in the Heisenberg box, a promising way to investigate the quality of detected components at each computed time-frequency point is to compare models inferred at adjacent time-frequency points in the Heisenberg box and examine whether a stable synchronization pattern arises. We call "pattern" the way channels are grouped together, meaning which channel is grouped with which other channels in the same component.

Let $M_{1}$ be the mixture model whose posterior probability has been inferred at a time-frequency location $\left[t_{1}, f_{1}\right]$, and $M_{2}$ a mixture model inferred at a neighbouring time-frequency location $\left[t_{2}, f_{2}\right]$. Neighbourhood $V_{M_{1}}$ of $M_{1}$ is defined by the Heisenberg box associated with the daughter wavelet $\psi_{a_{1}, \tau_{1}}(t)$ used to compute coefficients at $\left[t_{1}, f_{1}\right]$. This is justified by the fact that a large part of the surrounding daughter wavelets power overlaps in this region with the power of $\psi_{a_{1}, \tau_{1}}(t)$. The same kind of approach using redundancy of the CWT is used in [10], for single trial phase analysis in this case.

First, we compute a similarity measure $\mathcal{S}_{k_{1}, k_{2}}$ between each component $k_{1}$ from $M_{1}$ and each component $k_{2}$ from $M_{2}$ as follows:

$$
S_{k_{1}, k_{2}}=\frac{\sum_{c=1}^{C} q\left(z_{c, k_{1}} \mid M_{1}\right) q\left(z_{c, k_{2}} \mid M_{2}\right)}{\sqrt{\sum_{c=1}^{C} q\left(z_{c, k_{1}} \mid M_{1}\right)^{2}} \sqrt{\sum_{c=1}^{C} q\left(z_{c, k_{2}} \mid M_{2}\right)^{2}}}
$$

which is the cosine similarity [11] between vectors of posterior probability $q\left(z_{1, k_{1}}, \ldots, z_{C, k_{1}} \mid M_{1}\right)$ and $q\left(z_{1, k_{2}}, \ldots, z_{C, k_{2}} \mid M_{2}\right)$ that have been estimated previously. It compares the allocation of channels to the component $k_{1}$ in $M_{1}$ with the allocation of channels to the component $k_{2}$ in $M_{2}$. The measure $S_{k_{1}, k_{2}}$ takes values between 0 and 1 , reaching 1 if both components regroup the same channels and reject the same other channels, and 0 if their allocation is totally different.

Then we look for the best coupling between components of both model. A list of couples $L_{1,2}$ is formed iteratively, in a greedy approach : at each step the couple $\left(k_{1}, k_{2}\right)$ that maximizes $S_{k_{1}, k_{2}}$ is added to $L_{1,2}$, where $k_{1}$ and $k_{2}$ are not part of any previously selected couple.

The similarity $\mathbb{S}_{M_{1}, M_{2}}$ between $M_{1}$ and $M_{2}$ is computed as the average over the similarity measures between each couple in $L_{1,2}$ :

$$
\mathbb{S}_{M_{1}, M_{2}}=\frac{1}{\operatorname{card}\left(L_{1,2}\right)} \sum_{\left(k_{1}, k_{2}\right) \in L_{1,2}} S_{k_{1}, k_{2}}
$$

where card denotes the number of element of $L_{1,2}$

Components are considered effective only if they fulfil criterion (11). Consequently, when two models do not have the same number of effective components, noncoupled components $k$ are still added to $L_{1,2}$ as coupled with nothing $(k,$.$) and their similarity S_{k, .}$ value is null.

Finally, the stability of a model $M_{1}$ is computed as the average of the similarity of $M_{1}$ with all the other models contained in the time-frequency neighbourhood $V_{M_{1}}$ :

$$
\mathbb{S}_{M_{1}}=\frac{1}{\operatorname{card}\left(V_{M_{1}}\right)} \sum_{M_{2} \in V_{M_{1}}} \mathbb{S}_{M_{1}, M_{2}}
$$

\section{Results}

\subsection{Artificial dataset}

The method has first been evaluated with an artificial dataset made of transient waves buried in white Gaussian noise.

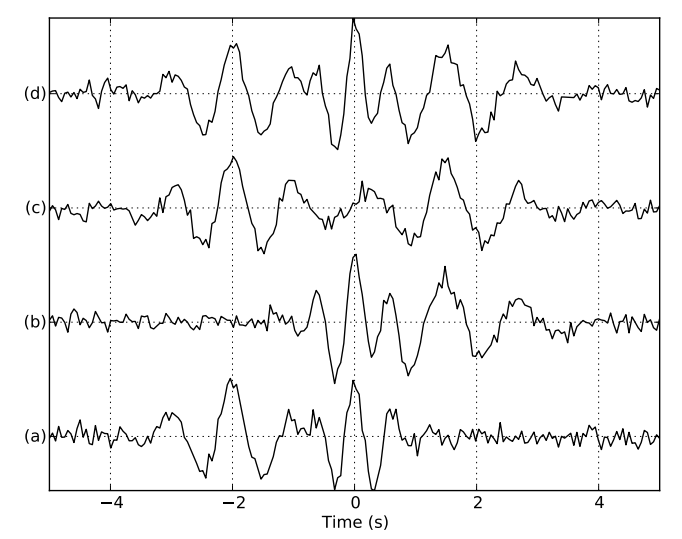

Figure 1: Time courses of the 4 types of channels contained in the artificial dataset. Types (a), (b) and (c) contain 2 of 3 Gabor atoms. Type (d) contains all 3 Gabor atoms.

This dataset consists in 40 channels. Each channel was composed by a set of Gabor atoms, defined using the 


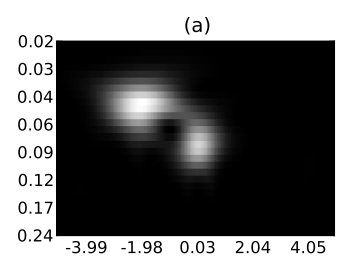

(c)
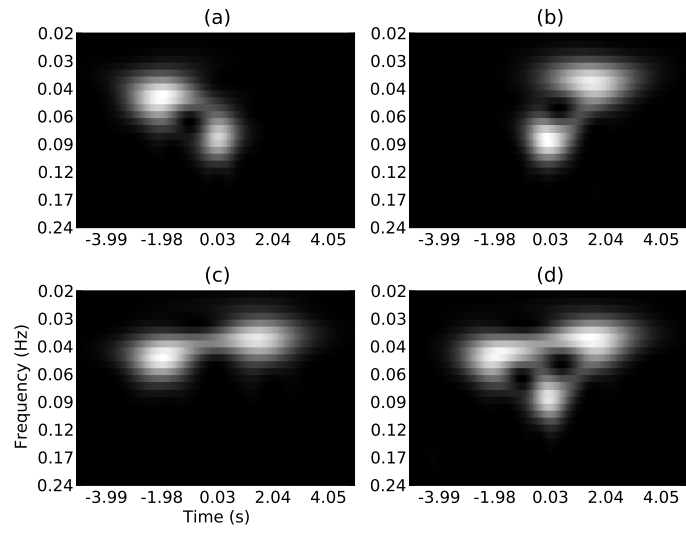

(d)

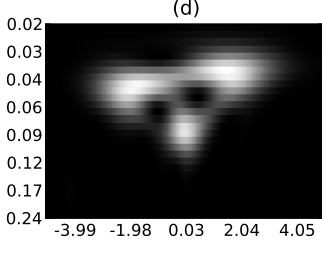

Figure 2: Squared modulus of the CWT computed with a normalized Morlet wavelet for the 4 types of channels contained in the artificial dataset. See figure 1 for the representation in the time domain.

normalized Morlet wavelet waveform (4). 3 Gabor atoms were used, with parameters $(\tau, a)=(0,0.5),(-2,0.8)$ and $(1.5,1.0) .10$ channels were generated for each possible pair of atoms, plus 10 channels with all three atoms. A Gaussian white noise with mean $\mu=0$ and standard deviation $\sigma=0.1$ is added to each channel. Sampling frequency is set at $1 \mathrm{~Hz}$.

For the first step of the method, the Morlet CWT, a geometrically sampled scale was used for the frequency axis [6]. Figures 1 and 2 illustrate the 4 possible types of channels with their associated representations in time and in the time-frequency plane.

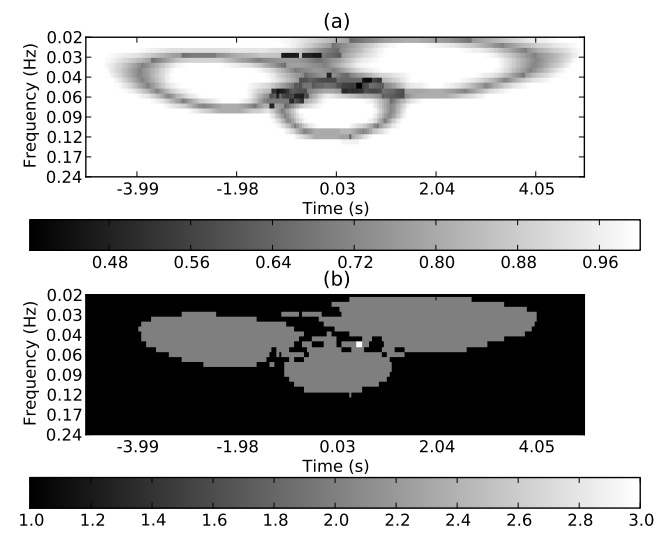

Figure 3: For the artificial dataset (a) Stability measure of each mixture model, at each time-frequency location. (b) Number of effective Gaussian components for each model

Results from the clustering and stability analysis steps are summarized in figure 3. The stability measure is the highest, close or equal to 1 , around the location of each Gabor atoms and outside their support region. The transition parts can be easily identified as region where the stability measure decrease. Besides, the number of effective components inferred within highly stable regions corresponds to the way channels are grouped:

- Outside Gabor atoms support region, all channels are gathered within one component as signal is just Gaussian noise.

- Near each Gabor atom center, 3 of the 4 types of channels gathered in one cluster, as these channels include this Gabor atoms, and the last type of channel forms the second cluster.

In the latter case, there is no misclassification of the channels, according to which Gabor atoms they are made of.

\subsection{Real dataset}

Experimental data were recorded with 17 bipolar microelectrodes implanted in behaving monkeys. Electrodes were placed in visual areas V1, V2, V4, the parietal area $\mathrm{A} 7$, the higher-level somato-sensoric area $\mathrm{A5}$, the post-central somato-sensoric area $\mathrm{A} 1$, the primary motor cortex A4, the pre-motor cortex A6. Monkeys had to perform a visuo-motor task: they had to move a lever according to some visual stimuli provided on a screen as moving sinusoidal grating. The full description of the dataset is done in [12]. For the present study, we restrict the data just to the first visual stimuli and response period.

Data were recorded at $1 \mathrm{kHz}$. We down-sampled it to $250 \mathrm{~Hz}$. Frequencies for the normalized Morlet CWT were chosen in order to focus the analysis on the gamma band activity, between $20 \mathrm{~Hz}$ and $70 \mathrm{~Hz}$.
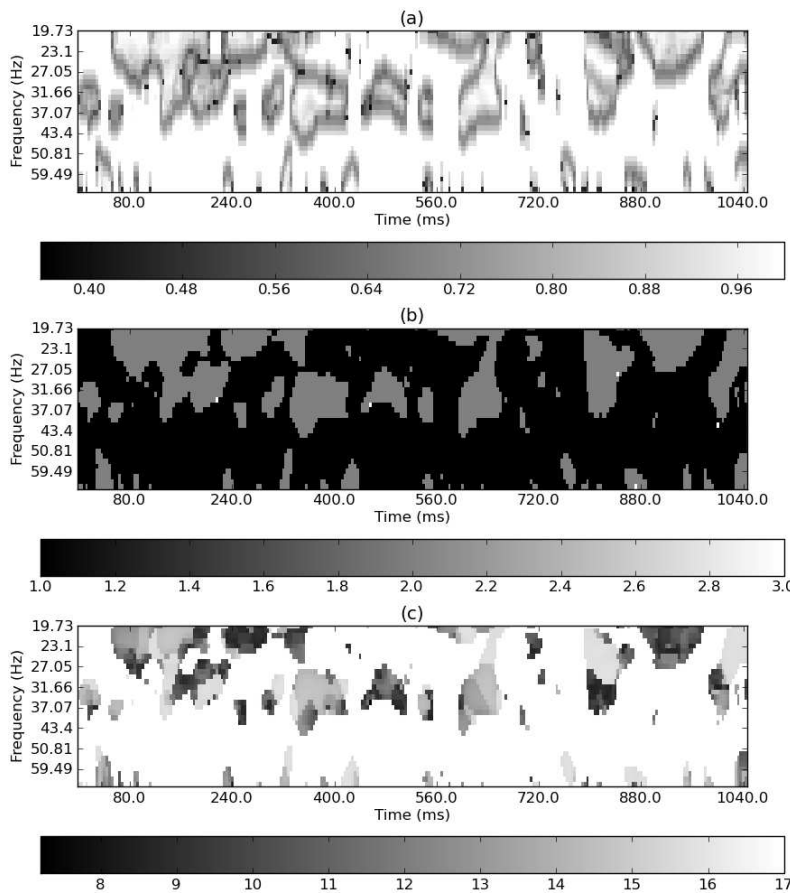

Figure 4: For one trial of the real dataset (a) Stability measure of each mixture model, at each time-frequency locations. (b) Number of effective Gaussian components for each model, (c) Maximum number of channels allocated to one of the components for each model

Figure 4 presents results on one arbitrary chosen trial. Within large time-frequency regions, associated models 
only have one effective component. It means the method did not manage to distinguish clear subsets of channels.

Most of the models with more than one effective component have two components. It is interesting to distinguish models which detect real subsets of channels from those that just reject one channel whose power is an outlier w.r.t the all of the other channels. This is illustrated by Figure 4 (c).

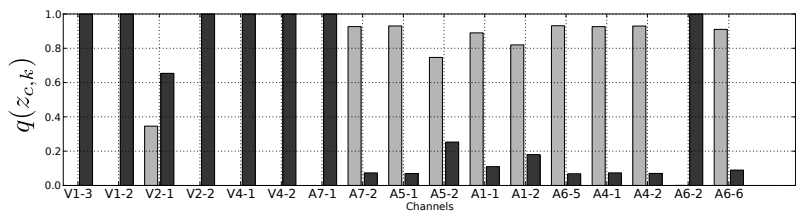

Figure 5: For the mixture model inferred at the timefrequency point $[804 \mathrm{~ms}, 34.3 \mathrm{~Hz}]$, posterior probability $q\left(z_{c, k}\right)$

Finally, combining information from figure 4 (a), (b) and (c), it is possible to select time-frequency regions of interest as those where models are stable, have more than one effective component and these components regroup more than one channel.

An example of a such region is around $[804 \mathrm{~ms}, 34.3 \mathrm{~Hz}]$. The inferred model at $[804 \mathrm{~ms}, 34.3 \mathrm{~Hz}]$ has a stability of 0.968 , and channels are distributed evenly between both effective components. Figure 5 illustrates the way channels are allocated to each of both components, through the posterior probability $q\left(z_{c, k}\right)$, which corresponds to the probability for each channel $c$ to belong to the Gaussian component $k$. Channels corresponding to motor areas are mostly allocated to one component whereas channels related to visual areas are allocated to the other component. Comparing the power represented by the mean of each Gaussian component, it is possible to say that visual areas are less synchronized at this time-frequency location than motor areas.

\section{Conclusion}

We have presented in this paper a new method to investigate ERS/ERD in multi-channels recordings of brain signals. This method can be used on single trials, without any baseline activity assumption. Due to the clustering approach used, it only distinguishes when subsets of recorded channels have different power at some time-frequency locations. Thus it cannot be used to investigate a global change of power within all channels.

Further developments will be focused on gathering single trials results, in order to study variability between trials.

\section{References}

[1] R. Q. Quiroga, A. Kraskov, T. Kreuz, and P. Grassberger, "Performance of different synchronization measures in real data: A case study on electroencephalographic signals," Phys. Rev. E, vol. 65, p. 041903, Mar 2002.
[2] J. Dauwels, F. Vialatte, T. Musha, and A. Cichocki, "A comparative study of synchrony measures for the early diagnosis of alzheimer's disease based on eeg," NeuroImage, vol. 49, no. 1, pp. 668-693, 2010 .

[3] G. Pfurtscheller and F. H. L. da Silva, "Event-related eeg/meg synchronization and desynchronization: basic principles," Clinical Neurophysiology, vol. 110, no. 11, pp. 1842-1857, 1999.

[4] S. Mallat, A Wavelet Tour of Signal Processing, Third Edition: The Sparse Way. Academic Press, 2008.

[5] I. Simonovski and M. Boltezar, "The norms and variances of the gabor, morlet and general harmonic wavelet functions," Journal of Sound and Vibration, vol. 264, no. 3, pp. 545-557, 2003.

[6] D. Jordan, R. W. Miksad, and E. J. Powers, "Implementation of the continuous wavelet transform for digital time series analysis," Review of Scientific Instruments, vol. 68, no. 3, pp. 14841494, 1997.

[7] C. M. Bishop, Pattern Recognition and Machine Learning (Information Science and Statistics). Springer, 2007.

[8] M. J. Beal, Variational Algorithms for Approximate Bayesian Inference. $\mathrm{PhD}$ thesis, Gatsby Computational Neuroscience Unit, University College London, 2003.

[9] J. Winn, Variational Message Passing and its applications. $\mathrm{PhD}$ thesis, University of Cambridge, Oct. 2003.

[10] J. Lachaux, E. Rodriguez, M. L. V. Quyen, A. Lutz, J. Martinerie, and F. J. Varela, "Studying singletrials of phase synchronous activity in the brain," International Journal of Bifurcation and Chaos, vol. 10, no. 10, pp. 2429-2439, 2000.

[11] P.-N. Tan, M. Steinbach, and V. Kumar, Introduction to Data Mining. Addison Wesley, 2005.

[12] A. Hutt and M. H. Munk, "Mutual phase synchronization in single trial data," Chaos and Complexity Letters, vol. 2, no. 2/3, pp. $225-246$, 2006. 